\title{
L'implantologie dans le champ juridique et déontologique 1. Les obligations du chirurgien dentiste
}

\author{
Implantology in the legal and ethical field \\ 1. The dental practitioner's obligations
}

HEDI ROMDANE, GILBERT DE MELLO

\begin{abstract}
RÉSUMÉ
Le chirurgien dentiste, dans sa relation avec les patients, est soumis non seulement aux règles morales élaborées par la vie sociale mais il doit également respecter les lois en vigueur. Parmi celles-ci, il y en a une qui le concerne directement : la responsabilité médicale. Les obligations liées à cette responsabilité connaissent une évolution importante.

L'implantologie bucco-dentaire est une discipline directement concernée par cette évolution, parce qu'elle se situe au carrefour de plusieurs disciplines. Elle fait appel à la fois à des connaissances en biologie, en parodontologie, en prothèse, en occlusion, en biomatériaux et même en informatique. Le praticien doit donc posséder une solide compétence dans ces domaines.

C'est dans ce contexte pluridisciplinaire que sa responsabilité est mise en cause. Les expertises sont donc devenues de plus en plus fréquentes en odonto-stomatologie, et en implantologie en particulier. Par ailleurs, outre ces obligations de moyens et de résultat, le praticien est tenu à l'information éclairée du patient, celuici étant devenu un acteur actif dans le processus de traitement. Désormais, sa responsabilité est engagée à tous les niveaux du processus de soin. Med Buccale Chir Buccale 2008 ; 14 : 85-95.
\end{abstract}

mots-clés : responsabilité, obligation, implantologie, capacité, compétence, faute, expertise.

médecine buccale chirurgie buccale

VOL. $14, \mathrm{~N}^{\circ} 2$ 2008

page 85

\section{SUMMARY}

As far as his relationship to the patient is concerned, not only has the dentist to respect the moral rules established by thousand years of community life, but he also has to play by the current laws. Among these, one concerns him directly: medical responsibility. The obligations associated with this responsibility are nowadays submitted to major changes.

Oral implantology is a field directly concerned by these changes because it stands at the crossroad of several fields. Indeed it requires knowledge in biology, periodontics, prosthesis, occlusion, biomaterials and even computer science in its in-depth knowledge. The practitioner has to prove solid skills in that field.

It is in this multidisciplinary context that his responsibility is challenged. The expertises have become more and more frequent in dentistry in general and in implantology in particular. In addition, beyond these obligations of means and results, the practitioner has to listen closely to the information provided by his patient who plays now an active role in the treatment process. Med Buccale Chir Buccale 2008 ; 14 : 85-95.

keywords: responsibility, obligations, implantology, ability, skill, malpractice, expertise. 
médecine

buccale

chirurgie

buccale

VOL. $14, \mathrm{~N}^{\circ} 2$ 2008

page 86
L'engouement suscité, ces dernières années, par la thérapeutique implantaire n'a fait qu'accroître le nombre d'échecs. Le manque de formation et l'incompétence de certains praticiens, peu scrupuleux, mais heureusement peu nombreux, se traduisent par une augmentation du nombre de plaintes déposé pour faute professionnelle, incriminant souvent des praticiens qui s'adonnent à l'implantologie sans formation suffisante. D'aucuns diront que l'évolution des mentalités est telle que nos patients ont tendance à calquer leurs pratiques sur celles des anglo-saxons qui n'hésitent pas à solliciter les hommes de loi pour résoudre des problèmes qui pourraient l'être par un dialogue basé sur la confiance et le respect mutuels. II est vrai qu'on ne peut s'empêcher de constater que dans la plupart des litiges qui arrivent chez juge, le manque de communication, voire l'incompréhension, sont souvent à l'origine de ces procédures judiciaires. II paraît cependant évident que la mise en cause de certains praticiens ne fait pas de doute.

L'implantologie ne s'improvise pas. C'est une discipline qui demande une solide connaissance théorique et une rigueur dans chaque étape de son déroulement. D'ailleurs, adresser un patient à un confrère compétent en implantologie lorsque le cas l'impose, ne constitue pas un aveu d'incompétence mais est au contraire un acte responsable.

Ce travail vise à relater les conséquences, quelquefois fâcheuses, d'un traitement implantologique et prothétique mal conçu et mal exécuté qui est soumis, par le truchement d'une procédure judiciaire, à un expert. Cette procédure réserve une place de choix au patient qui devient l'acteur central du dispositif. Chaque étape prend alors une importance particulière. Le praticien étant soumis à des exigences juridiques, déontologiques, et scientifiques, le moindre faux pas peut engendrer des conséquences pour le moins désagréables.

Mais alors, qu'attendent les patients dans le domaine de la dentisterie et en particulier celui de l'implantologie ? Et que peut-on trouver d'un point de vue juridique, qui puisse clarifier la relation entre le praticien et le patient demandeur de prothèse implanto-portée?
La réponse à ces questions ne peut être qu'une photographie instantanée de la législation compte tenu de l'évolution de la jurisprudence et de la déontologie. Pour conclure, on rappellera les moyens disponibles dans l'arsenal thérapeutique et déontologique pour satisfaire les exigences réglementaires et légales de l'exercice quotidien.

\section{LE CADRE LEGISLATIF ET JURIDIQUE DE LA PROTHESE IMPLANTO-PORTEE}

Pour être au diapason de la législation et des données acquises de la science, la pratique de la prothèse implanto-portée comporte certaines étapes indispensables que le thérapeute doit respecter pour accomplir sa mission.

La première de ces étapes est constituée par l'examen du patient candidat à la prothèse implanto-portée. Cet examen global préalable doit être méticuleux et exhaustif. II se décline en plusieurs points ${ }^{[1]}$ :

- Un questionnaire médical doit être soumis au patient afin de préciser ses antécédents médicaux : allergies, prise de médicaments, maladies dégénératives...

- Un examen clinique est réalisé pour détecter toute anomalie perceptible : insuffisance de hauteur des crêtes, parodontite avancée...

- Des examens radiologiques qui permettent de visualiser globalement la situation dentaire et la hauteur approximative d'os disponible dans la zone où la mise en place d'implants est envisagée. Des examens complémentaires seront demandés dans certains cas.

- Un examen du profil psychologique et social du patient. Est-il motivé pour accepter des séances de soins itératives et relativement lourdes ? A-t-il des doléances à formuler?

- Une prise d'empreintes d'étude qui, avec l'examen radiologique, va renseigner sur la faisabilité de la reconstitution prothétique.

- La réalisation d'un guide chirurgical si nécessaire.

- Une correspondance avec le chirurgien ou le prothésiste selon le cas pour une réflexion approfondie sur la conception globale de la prothèse implanto-portée. 
- L'élaboration d'un plan de traitement commun avec le consentement éclairé du patient.

- Et, en dernier ressort, la réalisation du traitement.

L'enchaînement de ces différentes étapes, en apparence simples, n'en est pas moins soumis à la rigueur qui s'impose pour tout acte thérapeutique. II n'est, en effet, pas rare, et les dossiers qui aboutissent chez le juge, le montrent régulièrement, que des aléas thérapeutiques, des erreurs d'exécution, voire des fautes, interfèrent avec le cheminement bien codifié du parcours thérapeutique. Les paramètres qui gravitent autour de sa réalisation sont nombreux, comme par exemple: les doléances du patient, son état de santé, le respect des rendez-vous, le seuil de tolérance de la douleur, l'état psychologique, l'environnement social, les incidents de parcours qui peuvent survenir lors de l'attribution des soins. Ces paramètres subjectifs sont autant d'éléments qui peuvent perturber la péréquation entre la bonne fin des travaux et leur conformité avec les données acquises de la science.

La vigilance est par conséquent de rigueur. Le praticien, ou plutôt les praticiens, en associant le praticien prothésiste, doivent veiller à la bonne exécution du travail faute de voir leur responsabilité engagée car ils sont soumis à des obligations précises sanctionnant la bonne fin des travaux. II est par conséquent intéressant d'étudier le champ des obligations à laquelle sont soumis les praticiens en odonto-stomatologie.

\section{LA LÉGISLATION}

S'il y a un domaine où la législation a beaucoup évoluée ces dernières années, et à une vitesse soutenue, c'est bien celle qui régit le domaine de la relation patient-praticien. Le patient s'est vu attribuer de nouveaux droits et se retrouve acteur à part entière dans la prise en charge de sa santé. Bien sûr, la décision médicale demeure l'affaire du professionnel mais celui-ci doit désormais apporter des informations au patient, qui est parfois intarissable sur les questions concernant sa santé. Cette soif de vouloir tout savoir, tout comprendre, doit être mise à profit pour renforcer le lien de confiance avec le patient. Ceci est primordial car, sans la confiance, un traitement quel qu'il soit ne peut aboutir à un résultat satisfaisant pour le patient. L'obligation d'informer comporte donc un côté positif.

C'est dans ce contexte législatif, d'aucuns disent dans ce carcan législatif, que le patient s'est vu doter d'outils juridiques pour faire face aux éventuelles fautes des professionnels de santé. Désormais, les actes professionnels sont assortis d'obligations dont le praticien ne peut s'affranchir sans mettre en jeu sa responsabilité ${ }^{[2-6]}$. Ces obligations sont de 3 ordres :

- Obligation de moyens,

- Parfois obligation de résultats,

- Surtout obligation d'information.

Parmi ces 3 types d'obligation, l'obligation d'information a pris une importance particulière car, son encadrement juridique étant récent, elle peut poser plus de problèmes d'appréciation.

Par ailleurs, on observe une évolution de la mentalité de nos contemporains se traduisant par un besoin immodéré de sécurité. Ceci aboutit à une responsabilité plus étendue pour le professionnel de la santé lors de la réalisation de ses actes ${ }^{[7,8]}$. On est bien loin de l'arrêt de la Cour de Cassation du 18 juin 1835 qui précise que : «les médecins sont responsables lorsque dans l'exercice de leurs fonctions ils causent un préjudice aux malades en ayant commis une faute évidente, une négligence ou une imprudence ». A cette époque, les praticiens ne sont soumis à aucune obligation, y compris celle d'informer [9] : il est seulement fait mention de préjudice découlant de soins. Progressivement, l'immunité juridique du praticien va être grignotée. Maintenant, il se voit dans l'habit d'un prestataire de service soumis à des obligations.

II devient, par conséquent, périlleux d'entamer un processus thérapeutique en faisant fi de ces obligations, laissant ainsi des outils à la disposition du patient au cas où un incident, un litige, voire un accident, surviendraient [10-13]. Dans ce contexte, le praticien doit connaître précisément ces obligations car il y va de sa responsabilité envers le patient ${ }^{[14,15]}$. médecine

buccale

chirurgie

buccale

VOL. $14, \mathrm{~N}^{\circ} 2$ 2008

page 87 


\section{Les différentes responsabilités}

Dans les faits, la responsabilité du praticien peut être envisagée aussi bien dans le cadre déontologique, voire disciplinaire, que dans le domaine juridique ${ }^{[16]}$ où elle peut relever du civil ou du pénal. Les responsabilités civile et pénale s'imposent à tout citoyen dans le domaine de la vie courante. Elles n'ont donc pas de spécificités particulières dans notre domaine professionnel. D'une façon générale, chacun sait ou doit savoir que la responsabilité civile régit les relations médicales entre le praticien et son patient, qu'elles soient contractuelles ou non contractuelles. Le législateur d'une part et la jurisprudence d'autre part sont les seuls garants des règles nécessaires à la conduite des procédures et au règlement des conflits. La responsabilité pénale est mise en jeu en cas d'infraction au code pénal. Elle est relative à des faits qualifiés par des termes tels que faux, fraude à la médecine buccale chirurgie buccale

VOL. $14, \mathrm{~N}^{\circ} 2$ 2008

page 88 sécurité sociale, ou encore violation du secret médical, etc. La responsabilité disciplinaire est, quant à elle, liée à tout ce qui entre dans le cadre déontologique dont le Conseil d'Etat est le seul garant. Dans ce domaine, il n'est pas inutile de rappeler deux situations qui trouvent un écho très particulier dans notre profession : ce sont les manquements à l'honneur et à la probité.

\section{Le contrat médical et les obligations qui lui sont imputées}

Obligation de moyens et de résultats, soins conformes aux données acquises de la science, voire aux données actuelles de la science [13], faute lourde ou virtuelle, erreur, maladresse, aléas, responsabilité prouvée ou présumée, etc. Tout ceci fait partie d'une liste non exhaustive des écueils auxquels peut être confronté le praticien [17]. Ces faits qui peuvent surgir à tout moment lors de l'activité professionnelle, sont-ils inéluctables ? La réponse dépend de plusieurs facteurs. Tout d'abord la compétence du praticien, puis le cadre juridique dans lequel il exerce, c'est-à-dire le code de déontologie et celui de la santé publique. Ceux-ci régissent notre profession. Ces règles évoluent pour s'adapter à la législation et à la jurisprudence. Ce sont ces dernières qui ont remodelé le cadre juridique de notre acti- vité, pour imposer, petit à petit, les différentes obligations qui doivent avant tout être considérées comme un rempart à tout jugement arbitraire.

\section{L'obligation de moyens}

Elle se définit parfaitement par les trois termes suivants : diligence, morale, technique. Ces termes ne sont pas étrangers à notre vocabulaire puisqu'ils se trouvent en bonne place dans le code de déontologie et qu'ils forment désormais la trame de la jurisprudence. Les juges les ont intégrés dans leurs attendus. Quelle est être leur définition au regard de notre profession?

La diligence : être diligent, selon le dictionnaire Le Robert, c'est être appliqué, assidu, attentif, soigneux, zélé. L'art. 27 du titre II du code de déontologie est explicite en la matière : «le chirurgien dentiste qui a accepté de donner des soins à un patient s'oblige :

- à lui assurer des soins éclairés et conformes aux données acquises de la science, soit personnellement, soit, lorsque sa conscience le lui commande, en faisant appel à un autre chirurgien dentiste ou à un médecin,

- à agir toujours avec correction et aménité envers le patient et à se montrer compatissant envers lui, - à se prêter à une tentative de conciliation qui lui serait demandée par le Président du Conseil départemental de l'Ordre en cas de difficultés avec un patient ».

Il est par conséquent normal d'accueillir le patient au cabinet avec respect et considération, d'autant plus que ce dernier se présente souvent confiant quant à la résolution de ses problèmes. Ceci implique une attitude loyale envers lui. Attitude définie, le plus souvent, par l'estime et le respect que nous lui portons. La morale est dans ce cas le prélude à toute relation de confiance. La morale : c'est en fait l'éthique, la probité qui regroupe conscience, intégrité, droiture, honnêteté, loyauté. Dans l'esprit de la loi, par morale, il faut entendre conforme aux mœurs ou tout simplement conforme aux règles qui régissent nos actes professionnels. Cette morale, à la base de toute relation humaine loyale, prend toute son importance quand il s'agit de prodiguer des soins. II est, par conséquent, de notre devoir d'avoir conscience de nos compétences et des limites, même si nous 
avons la capacité légale d'exercer dans tous les domaines de la dentisterie. La dérive mercantile qu'on observe en matière d'implantologie, est là pour le rappeler : il est en effet de notoriété publique que l'implantologie fait partie de ces disciplines où il existe une pléthore de propositions de formation, souvent plus attrayantes les unes que les autres. Ces propositions émanent aussi bien d'organismes de formation continue, de facultés d'odontologie que de fabricants vantant les mérites de leurs implants par le truchement d'un cours dans un cadre parfois idyllique. Seule une formation à la fois théorique et pratique, délivrée par des instituts reconnus et validée par nos pairs, justifie une rémunération à la hauteur de notre compétence. Les honoraires engendrés par les actes hors nomenclature ne sont alors que le reflet d'un savoir faire. La technique touche à la fois à la capacité et à la compétence. La capacité du praticien, de par le privilège octroyé par le diplôme de chirurgien dentiste d'une part, et par la loi d'autre part - notamment l'art. L373 du Code de la Santé publique qui précise que «l'art dentaire se définit par la pratique du diagnostic et du traitement des maladies des dents, de la bouche et des maxillaires »-, confère à ce dernier, en théorie, le droit de « tout faire » dans le cadre professionnel. Toutefois, elle ne lui délivre pas pour autant un blanc seing quant à sa compétence. II est essentiel de distinguer alors le droit et le fait. En règle générale, quelle que soit la capacité d'une personne, celle-ci devra toujours répondre de ses actes et de leurs conséquences en vertu des art. 1382.1101.1346.1371 du Code civil.

Acquérir la capacité par l'obtention d'un diplôme constitue souvent la clef pour accéder à une profession. C'est cette capacité qui permet, juridiquement, à chaque professionnel de parfaire ses connaissances théorique et pratique. Pour les chirurgiens dentistes, ce cadre légal correspond à la pratique et à l'enseignement; il recouvre le domaine de la bouche, des dents, des maxillaires et des tissus attenants. Et même dans ce chemin bien balisé, chaque praticien doit connaitre ses propres limites et ne pas hésiter à prendre l'avis d'un tiers ou adresser son patient à un confrère plus qualifié. La compétence prend le pas sur la capacité. II faut avoir à l'esprit qu'en cas de dom- mages réels ou supposés, le patient peut s'adresser au juge. Celui-ci tient compte exclusivement de la compétence du professionnel qu'il fera évaluer par un expert, et non plus de la capacité professionnelle puisque celle-ci ne souffre pas d'ambiguiité dans la mesure où elle est concrétisée par un diplôme reconnu juridiquement. C'est là que le juriste et le chirurgien dentiste entrent en opposition. Pour le premier, le chirurgien dentiste a l'obligation de donner les meilleurs soins possibles au patient, autrement dit, il doit faire preuve de compétence. A cela, le second ne peut qu'afficher sa diligence pour des actes et soins consciencieux, attentifs et conformes aux données acquises de la science médicale grâce à une formation continue. C'est pourquoi les magistrats demandent souvent à l'expert de dire si les soins ont été prodigués selon «les données acquises de la science médicale » ou avec la terminologie consacrée par la loi Kouchner du 4 mars 2002, selon les « données médicales avancées 》 [8]. Comment peut-on définir les thérapeutiques conformes aux « données médicales avancées »? Est-ce que l'expression « les données médicales avancées » recèle, dans sa signification la plus étendue, aussi bien les « données acquises " que les « données actuelles » ? II semble qu'il y ait une distinction à faire entre les deux termes. C'est une distinction qui repose sur une nuance et la validation d'une donnée par l'expérimentation prend tout son sens. Une donnée acquise est une donnée qui a passé l'épreuve de l'expérimentation alors qu'une donnée actuelle est en attente ou en cours de validation. Ce exercice d'ordre sémantique n'est pas superflu car la notion de « données médicales avancées » qui, pour les juges, signifie "données acquises de la science ", est souvent associée à la notion de « recommandations de bonne pratique ". Cette relation étroite entre ces deux formules a été confirmée par Pierre Sargos en $1998{ }^{[4]}$ qui conclut « que les références médicales opposables (RMO), comme les recommandations de bonne pratique, ne peuvent être par principe, que la traduction des données acquises de la science ". Pour mémoire, la notion de « données acquises de la science » a fait son apparition avec l'arrêt Mercier en 1936, qui précise qu'« il se forme entre le médecin et son client médecine

buccale

chirurgie

buccale

VOL. $14, \mathrm{~N}^{\circ} 2$ 2008

page 89 
médecine

buccale

chirurgie

buccale

VOL. $14, \mathrm{~N}^{\circ} 2$ 2008

page 90 un véritable contrat comportant pour le praticien, l'engagement, sinon bien évidemment de guérir le malade, ce qui n'a d'ailleurs jamais été allégué, du moins de lui donner des soins, non pas quelconques (...) mais consciencieux, attentifs et, réserve faite de circonstances exceptionnelles, conformes aux données acquises de la science». Les données acquises de la science sont régulièrement rappelées par différents moyens : livres et traités médicaux, recommandations établies par des spécialistes ou des experts [19].

\section{L'obligation de résultat}

En matière de conception et de confection d'un appareillage prothétique, l'obligation de résultat est désormais opposable à la suite de l'arrêt du 23 novembre 2004 [20] de la Cour de Cassation qui dispose : « le chirurgien dentiste est, en vertu du contrat le liant à son patient, tenu de lui fournir un appareillage apte à rendre le service qu'il peut légitimement en attendre, une telle obligation, incluant la conception et la confection de cet appareillage, étant le résultat ». Cette décision qui concerne le produit et non sa conception reste critiquable puisqu'elle est contraire à la loi du 4 mars $2002{ }^{[8]}$ qui subordonne la responsabilité à l'existence d'une faute. II y a lieu de s'interroger sur cette décision qui, par extrapolation, pourrait justifier l'intitulé «obligation commerciale » de ce chapitre, tant le parallèle entre le concept médical et le concept commercial est de plus en plus d'actualité.

Obtenir un résultat, c'est effectivement un objectif que le patient et le praticien définissent d'un commun accord et à l'avance. Depuis la loi du 19 mai 1998 [21] qui transpose la directive européenne dans le droit français (art. 1386 du Code civil), les produits de la santé sont assimilés à des produits de consommation et, en tant que tels, leur défaut, s'il produit un quelconque dommage à «l'usage », engage la responsabilité du producteur (le praticien) de plein droit. Déjà la Cour de Cassation dans son arrêt du 6 juillet 1994 [22] avait estimé que les personnes avec lesquelles un médecin conclut un contrat médical doivent être considérées comme " consommateurs des services fournis par ce dernier ». Cet aspect est confirmé par la loi Kouchner du 4 mars 2002 qui vise les actes médicaux et les infections nosocomiales mais écarte les produits de santé (art. L1142-1 du Code de la Santé publique). II est désormais acquis que le professionnel de santé, prescripteur et fournisseur de matériaux (implants, prothèses, etc.), fait partie d'une chaîne commerciale de distribution : fabricant - médecin-distributeur - patient-usager. Or dans le domaine commercial, et dans le but de sécuriser le consommateur, le législateur considère qu'en cas de litige, c'est à la victime d'exposer le dommage causé par un produit défectueux et c'est au producteur (ou au distributeur) de prouver l'absence de défectuosité du produit. Cette obligation reste conscrite au produit lui-même. La garantie ne porte pas sur la performance de celui-ci mais uniquement sur la qualité de sa fabrication et les dommages qu'il est éventuellement susceptible d'engendrer [3]. Parmi les praticiens visés par cette possibilité de défectuosité des produits utilisés, on trouve les chirurgiens orthopédistes (prothèse du genou...), les chirurgiens plasticiens (prothèses mammaires...), les cardiologues (valves, stents, pacemakers...) et les odontologistes (implants, prothèses dentaires...). Pour pallier à ces désagréments et opérer en toute quiétude, il est indispensable de suivre les directives européennes $n^{\circ} 90-385 / C E E$ [23] pour les dispositifs médicaux implantaires et la directive $n^{\circ}$ 93-42/CEE ${ }^{[24]}$ pour les autres dispositifs avec leur réglementation stricte relative à la commercialisation des biens médicaux qui ne doivent pas compromettre « l'état clinique et la sécurité des patients et utilisateurs 》 [25]. Le marquage Communauté Européenne (CE) est là pour matérialiser la conformité à ces exigences. Les implants et les prothèses dentaires sont ainsi marquées du sceau de la CEE [25]. Le label de qualité AFNOR et l'attestation de conformité aux performances exigées par l'AFSSAPS (art. 5211-2 du Code de la Santé publique) ${ }^{[26]}$ sont autant de garanties préservant de tout dommage susceptible de nuire aux patients. II n'empêche que ces derniers peuvent toujours invoquer l'insatisfaction au regard d'une restauration prothétique et se présenter devant un tribunal pour ce seul motif, témoins les arrêts de la Cour de Cassation du 17-2-71 [27] et du 15-11-97 [28]. Ils viennent confirmer cette obli- 
gation qui ne repose que sur des critères subjectifs, utilisés souvent par les mauvais payeurs.

\section{L'obligation d'informer le patient et de recueillir son consentement}

La notion de consentement ou de consentement éclairé du patient a été exposée et développée lors du procès de Nuremberg en 1947, dans le volet traitant les exactions des médecins nazis, où il est dit qu'« il est absolument essentiel d'obtenir le consentement éclairé et volontaire du malade ". C'est une obligation pré-contractuelle qui sert à définir le cadre dans lequel le praticien et son patient sont tenus d'évoluer de concert dans l'application du plan de traitement en toute connaissance de cause [21]. Ce statut d'information du patient a été défini en 1906, puis en 1970 en ces termes: «ll s'agit de transmettre la capacité technique d'un praticien à son patient, de donner des informations à celui qui ne sait pas » [29]. Le droit du patient à l'information en vue de donner son consentement est désormais essentiel et forme la pièce maîtresse des rapports entre praticien et patient ${ }^{[30]}$.

\section{La nature de l'information à donner au patient}

Peut-on définir la nature de l'information ? Par quel canal doit-elle cheminer ? Comment s'assurer qu'elle soit bien comprise ? Pour répondre à ces questions, on dispose de deux sources de données : la jurisprudence et le code de déontologie. L'arrêt de la Cour de Cassation du 29 mai $1951^{[31]}$ stipule que «le contrat qui se forme entre le praticien et son client comporte, en principe, l'obligation pour le praticien de ne pratiquer aucune intervention sans avoir au préalable obtenu l'assentiment du malade". Bien que cette obligation disparaisse en cas d'urgence ou de danger immédiat, comment s'assurer dans la pratique quotidienne que l'information donnée est bien comprise par le patient ? ${ }^{[32]}$. Chaque patient ayant sa propre personnalité, il faudra tenir compte :

- De l'aspect humain : pour s'assurer que le patient accueille l'information sur la réalité de son état avec calme et pondération ;

- De l'aspect juridique, car nous assistons à un télescopage entre ce qui relève de la loi et ce qui se heurte à la conscience dans l'attribution de l'information ;
- De l'aspect matériel car il faut définir un support pour concrétiser l'information donnée au patient et d'en conserver la trace pour pouvoir en justifier la réalité.

Informer le patient est une obligation qui a pris corps, en même temps que d'autres dispositions éparses préexistantes, dans le Code de Santé publique et le Code de Déontologie, après la promulgation de la loi du 4 mars 2002 [8,33]. Celle-ci a quelque chose de particulier car elle instaure le renversement radical des rôles dans l'établissement de la preuve entre le patient et le praticien. En effet, en 1995, la Cour de Cassation n'a pas hésité à décider qu'il appartenait au praticien soignant de faire la preuve de l'accomplissement de son devoir d'informer. La preuve de l'information qui était à la charge du patient est désormais l'affaire du praticien. Jusqu'en 1997, le patient était tenu de prouver qu'il n'avait pas été informé par son praticien des risques de l'intervention. Cette preuve étant souvent bien difficile à apporter, le patient était par conséquent débouté de ses prétentions en matière de dommages et intérêts. Ils'agit d'un point essentiel bien que l'information du patient reste une entité très relative et assez floue. Le praticien est désormais incité à clarifier la situation et à utiliser des repères légalement et juridiquement reconnus afin de préserver sa responsabilité dans sa relation contractuelle avec le patient. En effet, entre les 2 acteurs, il faut parler de contrat et non plus d'attribution de soins [34]. Ce contrat est certes un contrat médical mais son contenu lie deux sujets pour un même objectif en parfaite connaissance de cause. En cela, il n'est pas différent d'un autre. Pour être valide, ce contrat doit, par conséquent, obéir à certaines conditions légales, à savoir : la capacité des parties, un objet certain, déterminé, possible et licite, une cause certaine et licite, le consentement des parties. Ces différents points définissant le contrat pourraient paraître d'une grande banalité s'il ne s'agissait d'un domaine qui touche à l'intégrité de l'individu. D'où, au delà de ce contrat, somme toute ordinaire, la dimension importante que prend le consentement parfaitement éclairé du patient à subir des soins, en confiant "son corps" au praticien. Si le corps subit des dommages involontaires par la faute du praticien, celui-ci doit médecine

buccale

chirurgie

buccale

VOL. $14, \mathrm{~N}^{\circ} 2$ 2008

page 91 
médecine

buccale

chirurgie

buccale

VOL. $14, \mathrm{~N}^{\circ} 2$ 2008

page 92 en subir les conséquences. C'est donc une forme de protection du profane (patient) face au savant (praticien) que le droit tente d'établir. Le praticien doit informer son patient de tout risque, si minime soit-il, lié à l'intervention qu'il doit subir, même si ce risque est exceptionnel.

La matérialisation de cette information doit être classée de façon à favoriser la continuité des soins. C'est ce qui ressort des recommandations de l'Agence Nationale de l'Accréditation et d'Evaluation en Santé (l'ANAES) d'avril 2000 [35]. Lorsque le traitement est réalisé par plusieurs praticiens de différentes spécialités, chacun d'eux doit informer le patient à son niveau de compétence. L'article de la loi du 4 mars 2002 du Code de la Santé publique est explicite : " cette information incombe à tout professionnel de santé dans le cadre de ses compétences et dans le respect des règles professionnelles qui lui sont applicables $»\left[{ }^{[8]}\right.$.

\section{- Les différents types d'information}

L'information est donnée pour éclairer le patient sur la nature de son affection et le traitement qu'on lui propose avec tout son cortège de difficultés ou de risques ${ }^{[9]}$.

En respectant le principe selon lequel l'information doit être appropriée à l'état du patient, il paraît évident que la rédaction d'écrits pour se constituer des preuves que l'information a été donnée ne peut tenir lieu de règle absolue ${ }^{[12]}$. Les recommandations de l'ANAES, qui ont désormais valeur légale, représentent une aide. Ces recommandations de bonne pratique qui ont été homologuées par un arrêté du Ministre chargé de la Santé définissent les modalités qui valident l'information. Celle-ci doit être nécessairement transmise oralement, car c'est sous cette forme qu'elle peut être adaptée à chaque patient et à sa capacité de compréhension ; le praticien doit donc trouver le temps et la disponibilité indispensables à cette information. Selon le code de Santé publique, cette information doit également être délivrée au cours d'un entretien individuel.

Cette information sous forme orale n'exclut pas une information écrite. Celle-ci ne peut être qu'un complément mais n'est pas une obligation; de surcroît, cet écrit n'a pas vocation à recevoir la signature du patient. II ne doit être assorti d'au- cune formule obligeant le patient à y apposer sa signature. De plus, ces recommandations précisent que l'information écrite ne peut pas être adressée par courrier, ni par fax, ni par courriel car le praticien ne peut être sûr de la réception de la lettre par son destinataire. Il est par conséquent indispensable que l'information soit donnée à l'occasion d'un face à face physique.

- L'information du patient et le secret médical Le secret médical représente un principe intangible. Comment alors délivrer l'information sans trahir le secret médical ? Trois cas de figure peuvent se présenter. La juridiction les a bien mis en exergue au fil du temps.

Le patient mineur en état de comprendre doit être informé. Cependant, le Code civil distingue, deux situations. Dans la première, l'art. 372.2 relatif à l'autorité parentale prévoit que pour les actes usuels, un parent peut accomplir seul un de ces actes, l'accord de l'autre étant présumé acquis ; l'art. 42 stipule que l'information du mineur en état de comprendre et de supporter l'information est une obligation. La deuxième situation est relative au mineur : celui-ci peut donner son avis dont il sera tenu compte dans toute la mesure du possible. Partant de là, et par dérogation au principe de l'autorité parentale, la loi autorise le mineur à s'opposer à l'information de ses parents et à garder le secret sur son état de santé lorsque le traitement, voire l'intervention, devient une nécessité vitale pour sauvegarder sa santé. II doit être fait mention écrite dans le dossier médical, de l'opposition du mineur à l'information de ses parents. Cette situation rend difficile la position du praticien. Celui-ci doit bien analyser le contexte car la demande du mineur peut traduire l'expression d'un propos impulsif plutôt qu'un choix mûrement réfléchi.

Pour le majeur sous curatelle, son consentement est juridiquement valable pour des situations où sa santé exige des soins. Son pouvoir reste cependant limité puisque, pour un accord d'ordre patrimonial entre autres, il est impératif que le curateur soit saisi, par exemple l'accord pour un devis prothétique nécessite l'avis du curateur. Enfin, la personne de confiance : elle est désignée par le patient et il ne s'agit pas obligatoirement d'un 
proche. Dans cette situation nouvelle, le secret professionnel n'est plus respecter stricto sensu.

\section{- Les limites du patient et le secret médical}

II existe quelques situations où le praticien peut passer outre l'information du patient, certaines sont absolues, d'autres relatives. L'urgence est une situation absolue où l'information du patient n'est pas de mise. Cette situation est désormais légale mais sa définition reste floue. Les juges la définissent souvent par " nécessité absolue ", "nécessité évidente de l'intervention », " danger immédiat » ou encore « absence d'alternative thérapeutique et nécessité vitale » [22]. Le praticien doit par conséquent apprécier en conscience la situation. Un panel d'indices l'aide à s'orienter et à décider de l'urgence ou non mais cela peut être sujet à caution : parfois une certaine subjectivité peut fausser le jugement du praticien et le conduire à se méprendre. Les indices suivants sont à prendre en considération, avec précaution et objectivité : - Le lieu du dommage : domicile, hôpital, lieu de travail ;

- Le siège de la lésion : urgence organique, urgence psychologique ;

- L'âge du patient ;

- Le type de douleur provoquée.

Le refus du patient d'être informé constitue une situation relative qu'il faut analyser avec précaution car la responsabilité du praticien peut être mise en jeu. Selon la jurisprudence, « la volonté d'une personne d'être tenue dans l'ignorance d'un diagnostic ou d'un pronostic grave doit être respectée, sauf lorsque des tiers sont exposés à un risque de transmission » [24].

Dans ce cas de figure, on doit faire une remarque: l'art. 35 du Code de Déontologie donne la possibilité au praticien de passer outre son obligation d'informer, en cas de pronostic grave ou fatal pour son patient, tandis que c'est la loi qui permet au patient dans le même cas de figure de passer outre son droit d'être informé. Le médecin est alors libre de prodiguer les soins nécessaires mais un refus écrit du patient est exigé car celui-ci renonce à un droit. L'impossibilité d'informer représente une autre situation relative. Elle a été envisagée par la jurisprudence mais elle n'est pas très explicite sur la démarche conseillée au pra- ticien dans de telles situations. D'autres situations autorisent également le praticien à passer outre son devoir d'informer : malades sourds et muets, malades analphabètes, malades étrangers. Encore que d'aucuns pourraient s'interroger sur la possibilité matérielle d'informer ces patients. En effet, dans ces cas, ne peut-on pas faire passer l'information par écrit pour les sourds-muets, verbalement pour les analphabètes, par un traducteur pour les étrangers.

Indépendamment de ces situations où la décision du praticien d'informer le patient ou de ne pas l'informer est prise sans grandes difficultés, il en est d'autres où l'information ne peut être délivrée d'une façon appropriée. II s'agit des cas suivants: malade dans le coma, modification subite de l'option thérapeutique au cours d'une intervention, urgence au cours des situations précédentes. Quant aux limites d'ordre thérapeutique de l'information du patient, elles sont bien définies dans l'art. $35 \mathrm{du}$ Code de Déontologie médicale qui prévoit que «dans l'intérêt du malade et pour des raisons légitimes que le praticien apprécie en conscience, un malade peut être tenu dans l'ignorance d'un diagnostic ou d'un pronostic graves ". Dans le même esprit, la Cour de Cassation, dans sa décision en date du 23 mai 2000 [36], a pris soin de préciser que l'intérêt du malade devait s'apprécier en fonction de 3 critères : la nature de la pathologie, son évolution prévisible et la personnalité du malade.

Sur le plan juridique, la prudence s'impose car, eu égard à la subjectivité de ces critères et de surcroît leurs caractères d'exception, le praticien doit être toujours en mesure de justifier son choix en conservant des preuves (notes, échanges de courriers entre confrères, témoignages de membres de la famille).

\section{- L'information et ses objectifs essentiels}

L'information, pour être valide au regard de la loi, doit porter sur les points essentiels qui touchent au plus profond de la vie matérielle, sociale et psychique du patient. Les recommandations de I'ANAES [35], dans l'art. 1111-2 alinéa 1 découlant de la loi sur les droits des malades insistent sur les 8 points suivants :

- L'état de santé du patient : c'est ainsi que dans le cas d'un diagnostic ou d'un pronostic graves médecine

buccale chirurgie buccale

VOL. $14, \mathrm{~N}^{\circ} 2$ 2008

page 93 
médecine

buccale

chirurgie

buccale

VOL. $14, \mathrm{~N}^{\circ} 2$ 2008

page 94 "souvent une information brutale a de grandes chances d'être nuisible, voire dangereuse, même chez le mieux armé contre l'adversité » (L. René), mais les proches doivent être prévenus.

- Dans le cas où il y a refus de soins, il est du devoir du praticien d'éclairer le patient sur ce qui le menace s'il se soustrait aux soins.

- Dans les différentes investigations, traitements ou actions de préventions proposées, le praticien doit fournir des explications claires, c'est-à-dire aisées, faciles à comprendre, simples.

- Les alternatives aux techniques de soins proposés, si elles existent, doivent figurer sur le devis. - L'utilité et l'urgence éventuelle des soins déterminent le délai de réflexion du patient. Ce délai est une notion importante. Il conditionne la prise de décision tant au regard des soins proprement dits qu'au regard d'un devis prothétique.

- Dire leurs conséquences ou leurs risques fréquents ou graves normalement prévisibles.

- Mentionner les précautions générales et particulières recommandées au patient ainsi que les signes d'alerte détectables par le malade lui même. - Le coût et les modalités de prise en charge des soins envisagés : un jugement en date du 18 novembre 1999 [37] émanant du Tribunal d'Instance de Saumur-en-Auxois, précise clairement que le devoir d'information ne se limite pas à des informations purement médicales mais s'étend à l'information sur la prise en charge financière de l'acte ou du traitement proposés.

\section{- L'information et ses paradoxes}

L'information du patient, acteur désormais à part entière de sa santé, soulève la question de la définition de l'acte d'informer. Celui-ci a pris beaucoup plus d'importance et il semble même conditionner la réussite de nos actes. Pourtant les soins sont affaire de professionnel et ce dernier est seul compétent. Pourquoi donc une telle obstination dans le devoir d'informer le patient? Est-ce que le patient est devenu, soudain, apte à décider des soins qu'exige son état, dans un domaine qui lui est étranger ? Est-il seulement capable de faire le bon choix dans l'orientation thérapeutique qui le concerne, voire d'imposer son veto pour telle ou telle méthode thérapeutique, sans en avoir les moyens intellectuels ?
En disséquant le verbe «informer», on constate que d'un point de vue sémantique, son premier sens recèle l'action de donner une forme, une structure, voire une signification. En réalité, informer quelqu'un de quelque chose c'est tout simplement le mettre au courant, l'aviser, l'avertir, l'éclairer, le prévenir, le renseigner pour ne citer que les verbes en rapport avec notre contexte professionnel. Informer notre patient c'est donc l'éclairer, surtout l'avertir sur ce qui pourrait survenir comme difficultés, ou complications éventuelles à la suite de soins qu'on s'apprête à lui prodiguer. On voit poindre le paradoxe de cette demande : on propose des soins à notre patient tout en l'avisant de leurs éventuels méfaits. Dans la pratique, le devoir d'information du patient relève souvent de la quadrature du cercle. Quelle est donc la place de la confiance, pourtant indispensable à toute relation patient-praticien, dans l'environnement rigide de l'attribution de l'information? Quelle responsabilité pour le soignant? II est responsable de la matérialisation de la preuve puisque la Cour de Cassation a posé comme principe intangible, que « celui qui est légalement ou contractuellement tenu d'une obligation particulière d'information doit rapporter la preuve de l'exécution de cette obligation ". Ce principe ne souffre pas d'ambiguïté. Le dilemme reste par conséquent entier : ou bien le praticien agit dans un climat de confiance avec son patient au risque de voir sa responsabilité engagée à la suite d'un éventuel incident s'il se soustrait à la signature d'un document relatant les risques de son intervention, ou bien il respecte les exigences légales, au risque de susciter chez son patient un état d'angoisse qui peut aboutir au refus d'une intervention nécessaire.

La Cour de Cassation a considérablement modifié le cadre juridique de la responsabilité médicale, avec l'obligation d'information et le consentement éclairé. II semble qu'un nouvel équilibre a été atteint sous le contrôle des magistrats, secondés par les experts, tant sur le contenu de l'obligation d'informer que sur l'administration de la preuve. Même si l'obligation d'informer n'a aucune relation avec les complications post-opératoires, elle prend maintenant souvent une autre dimension dans le rapport des experts : par 
exemple, dans l'arrêté de la Cour de Paris du 9 avril $1999{ }^{[38]}$ pour une intervention de chirurgie mammaire l'expert, tout en déplorant l'absence d'information par le praticien, conclut qu'il n'existe aucune relation entre l'absence d'information et les incidents multiples survenus dans les suites opératoires, mais il déplore néanmoins ce manquement.

La pratique professionnelle doit s'appuyer sur les dispositions évolutives du Code de Déontologie. II s'agit d'un paradigme aussi simple à suivre que rassurant dans sa finalité. Les odontologistes gagneront à se fonder sur ses dispositions qui imposent des devoirs mais des devoirs que les praticiens eux-mêmes ont définis et adaptés au fil des ans, se conformant du même coup à l'évolution progressive des mentalités. Le Code de Déontologie ou les Codes de Déontologie si l'on inclut le Code de Déontologie médicale, sont reconnus par les magistrats et les experts comme une valeur normative et constituent une référence. Il faut donc bien réaliser que l'on ne fait qu'accompagner «l'évolution de la société » et c'est là la base de toute obligation, et en particulier de celle d'informer. Celle-ci ne constitue qu'un des points qui reflètent la volonté émanant de la col- laboration institutionnalisée entre l'OMS (Organisation Mondiale de la Santé) [39] et de nombreuses organisations gouvernementales dans le cadre du programme « la santé pour tous pour le XXle siècle ". Celles-ci ont impulsé un principe de réglementation qui offre aux patients les moyens de participer à l'amélioration de leur santé par une meilleure compréhension de leurs problèmes de santé et de leur traitement. C'est là que le droit à l'information du patient trouve toute sa consistance.

Après avoir évoqué les responsabilités civile et pénale du praticien, les obligations liées au contrat médical (obligations de moyen, de résultat, voire de compétence) et l'obligation d'informer, on a pu constater que ces dispositions sont sans cesse remaniées par la jurisprudence. Certes, on doit toujours s'adapter mais cet investissement permet d'entretenir une évolution dynamique dans la profession et, par delà, une amélioration régulière de la qualité des soins prodigués aux patients.

NDLR. La deuxième partie avec la bibliographie paraitra dans le $n^{\circ} 3 / 2008$ de MBCB. Elle traitera des obligations spécifiques du chirurgien dentiste dans le domaine de l'implantologie. médecine

buccale

chirurgie

buccale

VOL. $14, \mathrm{~N}^{\circ} 2$ 2008

page 95 